\title{
VARIANCE OF SUMS IN ARITHMETIC PROGRESSIONS OF DIVISOR FUNCTIONS ASSOCIATED WITH HIGHER DEGREE $L$-FUNCTIONS IN $\mathbb{F}_{q}[t]$
}

\author{
CHRIS HALL, JONATHAN P. KEATING, AND EDVA RODITTY-GERSHON
}

\begin{abstract}
We compute the variances of sums in arithmetic progressions of generalised $k$-divisor functions related to certain $L$-functions in $\mathbb{F}_{q}[t]$, in the limit as $q \rightarrow \infty$. This is achieved by making use of recently established equidistribution results for the associated Frobenius conjugacy classes. The variances are thus expressed, when $q \rightarrow \infty$, in terms of matrix integrals, which may be evaluated. Our results extend those obtained previously in the special case corresponding to the usual $k$-divisor function, when the $L$-function in question has degree one. They illustrate the role played by the degree of the $L$-functions; in particular, we find qualitatively new behaviour when the degree exceeds one. Our calculations apply, for example, to elliptic curves defined over $\mathbb{F}_{q}[t]$, and we illustrate them by examining in some detail the generalised $k$-divisor functions associated with the Legendre curve.
\end{abstract}

\section{INTRODUCTION}

For a fixed integer $k \geq 2$ the generalized divisor function $d_{k}(n)$ denotes the number of ways of writing a (positive) integer as a product of $k$ factors:

$$
d_{k}(n)=\sum_{m_{1} \cdot m_{2} \cdots m_{k}=n} 1
$$

It is related to the $k$ th power of the Riemann zeta-function, defined by

$$
\zeta(s)=\sum_{n=1}^{\infty} \frac{1}{n^{s}}
$$

when $\operatorname{Re} s>1$, in that

$$
\zeta(s)^{k}=\sum_{n=1}^{\infty} \frac{d_{k}(n)}{n^{s}}
$$

for $\operatorname{Re} s>1$.

Define $\Delta_{k}(x)$ by

$$
\Delta_{k}(x):=\sum_{n \leq x} d_{k}(n)-\operatorname{Res}_{s=1} \frac{x^{s} \zeta^{k}(s)}{s}=\sum_{n \leq x} d_{k}(n)-x P_{k-1}(\log x)
$$

where $P_{k-1}(u)$ is a certain polynomial of degree $k-1$; see, for example, Tit86] Chapter XII.

The mean square of $\Delta_{2}(x)$ was computed by Crámer Cra22] for $k=2$, and by Tong [Ton56] for $k \geq 3$ (assuming the Riemann Hypothesis $(\mathrm{RH})$ if $k \geq 4$ ), to be

$$
\frac{1}{X} \int_{X}^{2 X} \Delta_{k}(x)^{2} d x \sim c_{k} X^{1-\frac{1}{k}}
$$

We are pleased to acknowledge support under EPSRC Programme Grant EP/K034383/1 LMF: L-Functions and Modular Forms. JPK is also grateful for support from a Royal Society Wolfson Research Merit Award and ERC Advanced Grant 740900 (LogCorRM).. 
for a certain constant $c_{k}$. Heath-Brown HB92 showed that $\Delta_{k}(x) / x^{\frac{1}{2}-\frac{1}{2 k}}$ has a limiting value distribution (for $k \geq 4$ one needs to assume $\mathrm{RH}$ ); it is non-Gaussian.

Our main focus will be on sums of divisor functions over arithmetic progressions

$$
\mathcal{S}_{d_{k}}(A)=\mathcal{S}_{d_{k} ; X ; Q}(A)=\sum_{\substack{n \leq X \\ n=A \bmod Q}} d_{k}(n)
$$

For the standard divisor function $(k=2)$, it is known that if $Q<X^{2 / 3-\epsilon}$ then

$$
\mathcal{S}_{d_{2}}(A)=\frac{X p_{Q}(\log X)}{\Phi(Q)}+O\left(X^{1 / 3+o(1)}\right)
$$

for some linear polynomial $p_{Q}$. This is due to unpublished work of Selberg. For recent work on the asymptotics of sums of $d_{3}$ over arithmetic progressions, see [FKM15] and the literature cited therein.

The variance $\operatorname{Var}\left(\mathcal{S}_{d_{2} ; X ; Q}\right)$ of $\mathcal{S}_{d_{2}}$ has been studied by Motohashi [Mot73], Blomer [Blo08], Lau and Zhao [LZ12], the result being [LZ12] (we assume $Q$ prime for simplicity):

(i) If $1 \leq Q<X^{1 / 2+\epsilon}$ then

$$
\operatorname{Var}\left(\mathcal{S}_{d_{2} ; X ; Q}\right) \ll X^{1 / 2}+\left(\frac{X}{Q}\right)^{2 / 3+\epsilon} .
$$

(ii) For $X^{1 / 2}<Q<X$,

$$
\operatorname{Var}\left(\mathcal{S}_{d_{2} ; X ; Q}\right)=\frac{X}{Q} p_{3}\left(\log \frac{Q^{2}}{X}\right)+O\left(\left(\frac{X}{Q}\right)^{5 / 6}(\log X)^{3}\right)
$$

where $p_{3}$ is a polynomial of degree 3 with positive leading coefficient.

For $k \geq 3$, Kowalski and Ricotta [KR14] considered smooth analogues of the divisor sums $\mathcal{S}_{d_{k} ; X ; Q}(A)$, and among other things computed the variance for $Q^{k-1 / 2+\epsilon}<X<Q^{k-\epsilon}$.

In the function field setting, the analogous problem was studied by Rodgers, Rudnick and the second and third authors KRRGR18. Let $\mathcal{M}_{n} \subset \mathbb{F}_{q}[t]$ be the set of monic polynomials of degree $n$ with coefficients in $\mathbb{F}_{q}$. For a polynomial $Q \in F_{q}[x]$ of degree at least 2 , and $A$ co-prime to $Q$, set

$$
\mathcal{S}_{d_{k}, n, Q}(A):=\sum_{\substack{f \in \mathcal{M}_{n} \\ f=A \bmod Q}} d_{k}(f)
$$

Then for $n \leq k(\operatorname{deg} Q-1)$ the variance of this sum is given by

$$
\lim _{q \rightarrow \infty} \frac{\operatorname{Var}_{Q}\left(\mathcal{S}_{d_{k}, n, Q}\right)}{q^{n} /|Q|}=I_{k}(n ; \operatorname{deg} Q-1)
$$

where $I_{k}(n ; \operatorname{deg} Q-1)$ is a certain matrix integral over the unitary group $U_{\operatorname{deg} Q-1}(\mathbb{C})$ - see KRRGR18 and (1.1.1) below. This integral can be evaluated in a number of ways. Some of the main results obtained in KRRGR18 are set out in Section 5 below. It follows from these, for example that, for the classical divisor function $d=d_{2}$, if $\operatorname{deg} Q \geq 2$ and $n \leq 2(\operatorname{deg} Q-1)$, then

$$
\lim _{q \rightarrow \infty} \frac{\operatorname{Var}_{Q}\left(\mathcal{S}_{d_{2}, n, Q}\right)}{q^{n} /|Q|}= \begin{cases}\operatorname{Pol}_{3}(n), & n \leq \operatorname{deg} Q-1 \\ \operatorname{Pol}_{3}(2(\operatorname{deg} Q-1)-n), & \operatorname{deg} Q \leq n \leq 2(\operatorname{deg} Q-1)\end{cases}
$$

where

$$
\mathrm{Pol}_{3}(x)=\left(\begin{array}{c}
x+3 \\
3
\end{array}\right)=(x+1)(x+2)(x+3) / 6 .
$$

For general $k$, the variance may be expressed in terms of a lattice-point count and is a piecewise polynomial function of degree $k^{2}-1$. 
The function-field expressions for the variance of the generalized divisor function lead immediately to conjectures in the standard number-field setting [KRRGR18]. The conjecture when $k=3$ has recently been proved by Rodgers and Soundararajan [RS17].

Our aim here is to extend the line of research reviewed above to arithmetic functions associated with other $L$-functions in the same way that the generalized divisor function is related to the Riemann zeta-function. Our function field results (which we will soon state below; Theorem 1.1.2 and Theorem 2.0.8) can be used to motivate predictions in the number field setting. In order to illustrate these predictions, we focus now on two representative examples: elliptic curve $L$-functions and the Ramanujan $L$-function.

Let $E / \mathbb{Q}$ be an elliptic curve of conductor $N$ defined over $\mathbb{Q}$. The associated $L$-function $F(s)$ will be denoted by $L(s, E)$ and is given by

$$
L(s, E)=\prod_{p \mid N}\left(1-a_{p} p^{-s}\right)^{-1} \prod_{p \nmid N}\left(1-a_{p} p^{-s}+p^{-2 s+1}\right)^{-1}
$$

where $a_{p}$ is the difference between $p+1$ and the number of points on the reduced curve mod $p$

$$
a_{p}=p+1-\# \tilde{E}\left(\mathbb{F}_{p}\right) .
$$

When $p \mid N, a_{p}$ is either $1,-1$, or 0 . In general, we have the Hasse bound on $a_{p},\left|a_{p}\right|<2 \sqrt{p}$, hence the product converges and gives an analytic function for all $\operatorname{Re}(s)>3 / 2$. The $L$ function of $E$ expands as

$$
L(s, E)=\sum_{n=1}^{\infty} a_{n} n^{-s}
$$

where

$$
a_{p^{e}}=p^{e}+1-\# \tilde{E}\left(\mathbb{F}_{p^{e}}\right) .
$$

and for $n=\prod_{i=1}^{r} p_{i}^{e_{i}}$ with $p_{1}, \ldots, p_{r}$ distinct primes

$$
a_{n}=a_{p_{1}^{e_{1}}} \cdots a_{p_{r}^{e_{r}}}
$$

For each positive integer $k$ consider the multiplicative function $d_{k, E}: \mathbb{Z} \rightarrow \mathbb{Z}$ given by

$$
d_{k, E}:=\prod_{n_{1} \cdots n_{k}=n} a_{n_{1}} \cdots a_{n_{k}} .
$$

Note that $d_{k, E}$ gives the coefficients in the Dirichlet series expansion

$$
L(s, E)^{k}=\sum_{n=1}^{\infty} d_{k, E}(n) n^{-s} .
$$

Our results in the function field setting are analogous to computing the variance of the sum of $d_{k, E}$ in arithmetic progressions Define the sum over arithmetic progressions

$$
S_{x, c, E}(A):=\sum_{\substack{n \leq x \\ n=A} \bmod c} d_{k, E}(n) .
$$

Our function field result (see Theorem 2.0.8) leads us to predict that for $x^{\epsilon}<c, \epsilon>0$, the following holds:

with

$$
\operatorname{Var}\left(S_{x, c, E}\right) \sim \frac{x}{\phi(c)} a_{k}(L(s, E)) \gamma_{k}\left(\frac{\log x}{2 \log c}\right)(2 \log c)^{k^{2}-1} .
$$

$$
\gamma_{k}(c)=\frac{1}{k ! G(1+k)^{2}} \int_{[0,1]^{k}} \delta_{c}\left(w_{1}+\ldots+w_{k}\right) \prod_{i<j}\left(w_{i}-w_{j}\right)^{2} d^{k} w
$$


with $\delta_{c}(x)=\delta(x-c)$ being the delta distribution translated by $c$, and $G$ is the Barnes $G$-function, so that for positive integers $k, G(1+k)=1 ! \cdot 2 ! \cdot 3 ! \cdots(k-1)$ !. Here $a_{k}(L(s, E))$ is an arithmetic factor that can be written explicitly by $(2.5 .9)$ in $\left[\mathrm{CFK}^{+} 05\right]$.

Note that we can detect the degree of the $L$-function in question as the coefficient of $\log c$.

Another example of a degree-two $L$-function is the Ramanujan $L$-function:

$$
L(s, \tau)=\prod_{p}\left(1-\tau(p) p^{-s}+p^{-2 s+11}\right)^{-1},
$$

where $\tau$ is the Ramanujan tau function $\tau: \mathbb{N} \rightarrow \mathbb{Z}$ defined by the following identity:

$$
\sum_{n \geq 1} \tau(n) q^{n}=q \prod_{n \geq 1}\left(1-q^{n}\right)^{24},
$$

where $q=\exp (2 \pi i z)$. Ramanujan conjectured (and his conjecture was proved by Deligne) that $|\tau(p)| \leq 2 p^{11 / 2}$ for all primes $p$, hence the product converges and gives an analytic function for all $\operatorname{Re}(s)>13 / 2$. The $L$ function associated with $\tau$ expands as

$$
L(s, \tau)=\sum_{n=1}^{\infty} \tau(n) n^{-s}
$$

For each positive integer $k$ consider the multiplicative function $d_{k, \tau}: \mathbb{Z} \rightarrow \mathbb{Z}$ given by

$$
d_{k, \tau}:=\prod_{n_{1} \cdots n_{k}=n} \tau\left(n_{1}\right) \cdots \tau\left(n_{k}\right) .
$$

Note that $d_{k, E}$ gives the coefficients in the Dirichlet series expansion

$$
L(s, \tau)^{k}=\sum_{n=1}^{\infty} d_{k, \tau}(n) n^{-s} .
$$

Again we are led to speculate that for $x^{\epsilon}<c, \epsilon>0$, if

$$
S_{x, c, \tau}(A):=\sum_{\substack{n \leq x \\ n=A} \bmod c} d_{k, \tau}(n)
$$

then the following holds:

$$
\operatorname{Var}\left(S_{x, c, \tau}\right) \sim \frac{x}{\phi(c)} a_{k}(L(s, \tau)) \gamma_{k}\left(\frac{\log x}{2 \log c}\right)(2 \log c)^{k^{2}-1} . .
$$

Where $a_{k}(L(s, \tau))$ is an arithmetic factor that can be written explicitly by (2.5.9) in $\left.\mathrm{CFK}^{+} 05\right]$.

We set out our main results below, but first, by way of illustration, we examine a specific example where the $L$-function in question take a relatively simple and explicit form, and can defined in a self-contained way.

1.1. Divisors associated to Legendre curve L-function. Let $q$ be a power of a prime $p$. Let $\mathcal{M} \subset \mathbb{F}_{q}[t]$ be the subset of monic polynomials and $\mathcal{I} \subset \mathcal{M}$ be the subset of irreducibles. For each $n \geq 1$, let $\mathcal{M}_{n} \subset \mathcal{M}$ and $\mathcal{I}_{n} \subset \mathcal{I}$ be the respective subsets of elements of degree $n$.

Suppose $q$ is odd, and let $E / \mathbb{F}_{q}(t)$ be the Legendre curve, that is, the elliptic curve with affine model

$$
y^{2}=x(x-1)(x-t) .
$$

Its $L$-function is given by an Euler product

$$
L\left(T, E / \mathbb{F}_{q}(t)\right)=\prod_{\pi \in \mathcal{I}} L\left(T^{\operatorname{deg}(\pi)}, E / \mathbb{F}_{\pi}\right)^{-1}
$$

where $\mathbb{F}_{\pi}$ is the residue field $\mathbb{F}_{q}[t] / \pi \mathbb{F}_{q}[t]$. 
Each Euler factor of $L\left(T, E / \mathbb{F}_{q}(t)\right)$ is the reciprocal of a polynomial in $\mathbb{Q}[T]$ and satisfies

$$
T \frac{d}{d T} \log L\left(T, E / \mathbb{F}_{\pi}\right)^{-1}=\sum_{m=1}^{\infty} a_{\pi^{m}} T^{m} \in \mathbb{Z}[[T]] .
$$

We regard the coefficients $a_{\pi^{m}}$ as values of the multiplicative function $f \mapsto a_{f}$ on $\mathcal{M}$ given by the coefficients of the Dirichlet series expansion

$$
L\left(T, E / \mathbb{F}_{q}(t)\right)=\sum_{f \in \mathcal{M}} a_{f} T^{\operatorname{deg}(f)} .
$$

Thus $a_{1}=1$, and if $f=\prod_{i=1}^{r} \pi_{i}^{e_{i}}$ with $\pi_{1}, \ldots, \pi_{r}$ distinct elements of $\mathcal{I}$, then

$$
a_{f}=a_{\pi^{e_{1}}} \cdots a_{\pi^{e_{r}}} .
$$

For each positive integer $k$, consider the multiplicative function $d_{k, \text { Leg }}: \mathcal{M} \rightarrow \mathbb{Z}$ given by

$$
d_{k, \operatorname{Leg}}(f)=\prod_{\substack{f_{1}, \ldots, f_{k} \in \mathcal{M} \\ f_{1} \cdots f_{k}=f}} a_{f_{1}} \cdots a_{f_{k}} .
$$

Equivalently, $d_{k, \text { Leg }}$ gives the coefficients in the Dirichlet series expansion

$$
L\left(T, E / \mathbb{F}_{q}(t)\right)^{k}=\sum_{f \in \mathcal{M}} d_{k, \operatorname{Leg}}(f) T^{\operatorname{deg}(f)} .
$$

It is easy to see that this is a generalization of the $k$ th divisor function: replace $L\left(T, E / \mathbb{F}_{q}(t)\right)$ by the zeta function $Z\left(T, \mathbb{A}^{1} / \mathbb{F}_{q}\right)$ so that $f \mapsto a_{f}$ becomes the constant function $f \mapsto 1$.

Let $c \in \mathcal{M}$ be square free and $\Gamma_{q}(c)=\left(\mathbb{F}_{q}[t] / c \mathbb{F}_{q}[t]\right)^{\times}$. For each $n \geq 1$ and $A \in \Gamma_{q}(c)$, consider the sum

$$
S_{k, n, c}(A):=\sum_{\substack{f \in \mathcal{M}_{n} \\ f \equiv A \bmod c}} d_{k, \operatorname{Leg}}(f) .
$$

Let $A$ vary uniformly over $\Gamma(c)$, and consider the expected value

$$
\mathbb{E}_{A}\left[S_{k, n, c}(A)\right]:=\frac{1}{\left|\Gamma_{q}(c)\right|} \sum_{A \in \Gamma_{q}(c)} S_{k, n, c}(A)
$$

and the variance

$$
\operatorname{Var}_{A}\left[S_{k, n, c}(A)\right]:=\frac{1}{\left|\Gamma_{q}(c)\right|} \sum_{A \in \Gamma_{q}(c)}\left|S_{k, n, c}(A)-\mathbb{E}_{A}\left[S_{k, n, c}(A)\right]\right|^{2} .
$$

These moments depend on $q$, so one can ask how they behave when we replace $\mathbb{F}_{q}$ by a finite

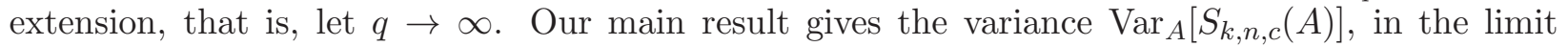
$q \rightarrow \infty$, in terms of a matrix integral. Let $U$ be an $R \times R$ matrix. Let std: $U_{R}(\mathbb{C}) \rightarrow \mathrm{GL}_{R}(\mathbb{C})$ be the representation given by the inclusion $U_{R}(\mathbb{C}) \subset \mathrm{GL}_{R}(\mathbb{C})$ and

$$
\wedge^{j} \operatorname{std}: U_{R}(\mathbb{C}) \rightarrow \mathrm{GL}_{R_{j}}\left(\wedge^{j} \mathbb{C}\right)
$$

be its $j$ th exterior power where $R_{j}=\left(\begin{array}{c}R \\ j\end{array}\right)$; we define $\left(\wedge^{j} \operatorname{std}\right)(g)=0$ unless $0 \leq j \leq R$. Define the matrix integrals with respect to Haar measure $d \theta$ on $U_{R}(\mathbb{C})$ the group of $R \times R$ unitary matrices

$$
I_{k}(n ; R)=\int_{U_{R}(\mathbb{C})}\left|\sum_{n_{1}+\cdots+n_{k}=n} \operatorname{Tr}\left(\left(\otimes_{i=1}^{k}\left(\wedge^{n_{i}} \mathrm{std}\right)\right)(\theta)\right)\right|^{2} d \theta .
$$

Then, as a special case of a general theorem we present in this paper, one can prove the following theorem: 
Theorem 1.1.2. If $\operatorname{gcd}(c, t(t-1))=t$ and if $\operatorname{deg}(c) \gg 1$, then

$$
\lim _{q \rightarrow \infty} \frac{\left|\Gamma_{q}(c)\right|}{q^{2 n}} \operatorname{Var}_{A}\left[S_{k, n, c}(A)\right]=I_{k}(n ; 2 \operatorname{deg}(c)-1)
$$

See Remark 2.0.9 for a brief explanation of how this follows from our general theorem. The factor 2 multiplying $\operatorname{deg}(c)$ corresponds to the degree of the Legendre curve $L$-function.

Corollary 1.1.3. In the case of $k=2$ we have for $n \leq 2 \operatorname{deg}(c)-1$

$$
\lim _{q \rightarrow \infty} \frac{\left|\Gamma_{q}(c)\right|}{q^{2 n}} \operatorname{Var}_{A}\left[S_{2, n, c}(A)\right]=\left(\begin{array}{c}
n+3 \\
3
\end{array}\right)
$$

and for $2 \operatorname{deg}(c)-1<n<4 \operatorname{deg}(c)-2$

$$
\lim _{q \rightarrow \infty} \frac{\left|\Gamma_{q}(c)\right|}{q^{2 n}} \operatorname{Var}_{A}\left[S_{2, n, c}(A)\right]=\left(\begin{array}{c}
4 \operatorname{deg} c-n+1 \\
3
\end{array}\right) .
$$

The Corollary will follow from evaluating the matrix integral in 95 .

\section{Statement of Main Theorem}

Let $\bar{K}$ be an algebraic closure of $K=\mathbb{F}_{q}(t)$ and $G_{K}$ be the Galois group $\operatorname{Gal}(\bar{K} / K)$. We regard $\mathcal{P}=\mathcal{I} \cup\{\infty\}$ as the set of places of $K$, and for each $v \in \mathcal{P}$, we write $\mathbb{F}_{v}$ for the residue field and $\operatorname{deg}(v)$ for its degree over $\mathbb{F}_{q}$. We also fix inertia and decomposition groups $I(v) \subset D(v) \subset G_{K}$ respectively and write $G_{v}$ for the quotient group $D(v) / I(v)$ and Frob $_{v} \in G_{v}$ for the Geometric Frobenius element.

For each finite subset $\mathcal{S} \subset \mathcal{P}$, let $K_{S} \subseteq \bar{K}$ be the maximal subfield which is unramified over $\mathcal{P} \backslash \mathcal{S}$ and $G_{K, \mathcal{S}}$ be the Galois group $\operatorname{Gal}\left(K_{S} / K\right)$. We abuse notation and write $I(v) \subseteq D(v)$ for the images of $I(v) \subset D(v) \subset G_{K}$ in $G_{K, \mathcal{S}}$ via the canonical quotient $G_{K} \rightarrow G_{K, \mathcal{S}}$. If $v \in \mathcal{S}$, then $I(v) \subseteq G_{K, \mathcal{S}}$ is isomorphic to $I(v) \subset G_{K}$, and otherwise $I(v) \subseteq G_{K, \mathcal{S}}$ is the trivial group.

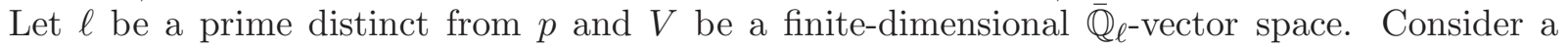
Galois representation

$$
\rho: G_{K, \mathcal{S}} \rightarrow \mathrm{GL}(V),
$$

that is, a continuous group homomorphism. For each $v \in \mathcal{P}$, let $V_{v}=V^{\rho(I(v))}$. We say that $s$ is the conductor of $\rho$ if $s$ is a square-free monic polynomial divisible by prime polynomials $v$ for which $V_{v}$ is strictly smaller than $V$. Let

$$
\rho_{v}: G_{v} \rightarrow \mathrm{GL}\left(V_{v}\right)
$$

be the composition of the restriction of $\rho$ to $D(v)$ and the quotient $D(v) \rightarrow G_{v}$, and let

$$
L\left(T, \rho_{v}\right):=\operatorname{det}\left(1-T \rho\left(\operatorname{Frob}_{v}\right) \mid V_{v}\right) .
$$

We attach several $L$-functions to $\rho$. One is the complete $L$-function and is given by the Euler product

$$
L(T, \rho):=\prod_{v \in \mathcal{P}} L\left(T^{\operatorname{deg}(v)}, \rho_{v}\right)^{-1} .
$$

Another is a partial $L$-function given by the Euler product

$$
L_{\text {fin }}(T, \rho):=\prod_{\pi \in \mathcal{I}} L\left(T^{\operatorname{deg}(\pi)}, \rho_{\pi}\right)^{-1}
$$

where now the Euler product is taken over the 'finite' places of $K$. If $L\left(T, \rho_{\infty}\right)$ is trivial, that is, if $V_{\infty}=0$, then these $L$-functions coincide, but in general

$$
L_{\text {fin }}(T, \rho)=\underset{6}{L(T, \rho) \cdot L\left(T, \rho_{\infty}\right) .}
$$


One reason for considering the partial $L$-function $L_{\text {fin }}(T, \rho)$ is that we can expand its Euler product as a Dirichlet series

$$
L_{\mathrm{fin}}(T, \rho)=\sum_{f \in \mathcal{M}} a_{f} T^{\operatorname{deg}(f)}
$$

where $f \mapsto a_{f}$ is the multiplicative function $\mathcal{M} \rightarrow \overline{\mathbb{Q}}_{\ell}$ given on prime powers by writing

$$
T \frac{d}{d T} \log \left(L\left(T, \rho_{\pi}\right)^{-1}\right)=\sum_{m=1}^{\infty} a_{\pi^{m}} T^{m} .
$$

Equivalently, for each $\pi \in \mathcal{I}$ and $m \geq 1$, we have

$$
a_{\pi^{m}}=\operatorname{Tr}\left(\rho_{v}\left(\mathrm{Frob}_{\pi}\right)^{m} \mid V_{v}\right)
$$

and if $f=\prod_{i=1}^{r} \pi_{i}^{e_{i}}$ is a prime factorization in $\mathbb{F}_{q}[t]$ with $\pi_{i} \neq \pi_{j}$ for $i \neq j$, then

$$
a_{f}=a_{\pi_{1}^{e_{1}}} \cdots a_{\pi_{r}^{e_{r}}}
$$

where $a_{1}=1$.

For each positive integer $k$, we define the $k$ th divisor function of $\rho$ as follows: it is the multiplicative function $d_{k, \rho}: \mathcal{M} \rightarrow \overline{\mathbb{Q}}_{\ell}$ given by

$$
d_{k, \rho}(f)=\prod_{\substack{f_{1}, \ldots, f_{k} \in \mathcal{M} \\ f_{1} \cdots f_{k}=f}} a_{f_{1}} \cdots a_{f_{k}}
$$

When $\rho$ is the trivial representation 1 (and thus $\operatorname{dim}(V)=1$ ), then this is the usual $k$ divisor function on $\mathcal{M}$ :

$$
d_{k, \mathbf{1}}(f)=\left|\left\{f_{1}, \ldots, f_{k} \in \mathcal{M}: f_{1} \cdots f_{k}=f\right\}\right|
$$

In general, we have the identity

$$
L_{\mathrm{fin}}(T, \rho)^{k}=\sum_{f \in \mathcal{M}} d_{k, \rho}(f) T^{\operatorname{deg}(f)}=\sum_{n=0}^{\infty}\left(\sum_{f \in \mathcal{M}_{n}} d_{k, \rho}(f)\right) T^{n} .
$$

Let $\mathcal{C} \subset \mathcal{P}$ be a finite subset containing $\infty$ and

$$
L_{\mathcal{C}}(T, \rho):=\prod_{\pi \notin \mathcal{C}} L\left(T^{\operatorname{deg}(\pi)}, \rho_{\pi}\right)^{-1}
$$

where $\pi$ runs over $\mathcal{I}$ and let

$$
R=\operatorname{deg}\left(L_{\mathcal{C}}(T, \rho)\right) .
$$

Thus $L_{\text {fin }}(T, \rho)=L_{\{\infty\}}(T, \rho)$, and in general,

$$
L_{\mathcal{C}}(T, \rho)^{k}=\sum_{n=0}^{\infty}\left(\sum_{\substack{f \in \mathcal{M}_{n} \\ \operatorname{gcd}(f, c)=1}} d_{k, \rho}(f)\right) T^{n}
$$

where $c \in \mathcal{M}$ is the product of all primes $\pi \in \mathcal{I} \cap \mathcal{C}$.

Let $\Gamma_{q}(c)$ be the finite abelian group $\left(\mathbb{F}_{q}[t] / c \mathbb{F}_{q}[t]\right)^{\times}$, and for each $A \in \Gamma_{q}(c)$, let

$$
S_{n, c, q}(A):=\sum_{f \in \mathcal{M}_{n}(A)} d_{k, \rho}(f)
$$

where

$$
\mathcal{M}_{n}(A):=\left\{f \in \mathcal{M}_{n}: f \equiv A \bmod c\right\}
$$


We regard $A$ as a random variable uniformly distributed over $\Gamma_{q}(c)$ and define its expected value

$$
\mathbb{E}_{A}\left[S_{n, c, q}(A)\right]:=\frac{1}{\left|\Gamma_{q}(c)\right|} \sum_{A \in \Gamma_{q}(c)} S_{n, c, q}(A)
$$

and variance

$$
\operatorname{Var}_{A}\left[S_{n, c, q}(A)\right]:=\frac{1}{\left|\Gamma_{q}(c)\right|} \sum_{A \in \Gamma_{q}(c)}\left|S_{n, c, q}(A)-\mathbb{E}_{A}\left[S_{n, c, q}(A)\right]\right|^{2}
$$

accordingly.

It follows easily the definition and (2.0.4) that

$$
L_{\mathcal{C}}(T, \rho)=\left|\Gamma_{q}(c)\right| \cdot \sum_{n=0}^{\infty} \mathbb{E}_{A}\left[S_{n, c, q}(A)\right] T^{n},
$$

so each expected value is the coefficient of an $L$-series. The main goal of this paper is to analyze the asymptotic behavior of $\left|\operatorname{Var}_{A}\left[S_{n, c, q}(A)\right]\right|^{2}$ as $q \rightarrow \infty$, that is, as we replace $q$ by a power $q^{r}$ and take $r \rightarrow \infty$. To do so, we must impose some hypotheses on $\rho$, e.g., we suppose that $\rho$ is punctually pure of weight $w$ (see section 6 in [HKRG17]). We also impose hypotheses on the Mellin transform of $\rho$, but before doing so we need some additional notation and terminology.

Let $\Phi_{q}(c)$ be the finite abelian group $\operatorname{Hom}\left(\Gamma_{q}(c), \overline{\mathbb{Q}}_{\ell}^{\times}\right)$. For each $\varphi \in \Phi_{q}(c)$, there is a corresponding Dirichlet character

$$
\varphi: G_{K, \mathcal{C}} \rightarrow \mathrm{GL}\left(\overline{\mathbb{Q}}_{\ell}\right)
$$

which we regard as a representation of $G_{K, \mathcal{R}}$ for $\mathcal{R}=\mathcal{C} \cup \mathcal{S}$ by composing $\varphi$ with the quotient $G_{K, \mathcal{R}} \rightarrow G_{K, \mathcal{C}}$. We also regard $\rho$ as a representation of $G_{K, \mathcal{R}}$ via the quotient $G_{K, \mathcal{R}} \rightarrow G_{K, \mathcal{S}}$, and we define the tensor-product representation

$$
\rho \otimes \varphi: G_{K, \mathcal{R}} \rightarrow \mathrm{GL}\left(V_{\varphi}\right)
$$

where $V_{\varphi}=V$ and $f \mapsto \rho(f) \varphi(f)$.

Let $L(T, \rho \otimes \varphi)$ and $L_{\mathcal{C}}(T, \rho \otimes \varphi)$ be the $L$-functions respectively defined by the Euler products in (2.0.1) and (2.0.2) with $\rho \otimes \varphi$ in lieu of $\varphi$. A priori each, of these is a power series with coefficients in $\overline{\mathbb{Q}}_{\ell}$, but Grothendieck showed both are rational functions in $\overline{\mathbb{Q}}_{\ell}(T)$, compare (1.4.7) of [Del80]. We say that $\varphi \in \Phi_{q}(c)$ is good for $\rho$ iff it lies in the set

$$
\Phi_{q}(c)_{\rho \text { good }}:=\left\{\varphi \in \Phi_{q}(c): L(T, \rho \otimes \varphi)=L_{\mathcal{C}}(T, \rho \otimes \varphi) \in \overline{\mathbb{Q}}_{\ell}[T]\right\},
$$

and otherwise we say that $\rho$ is bad for $\rho$.

The hypothesis that $\rho$ is punctually pure implies that both $L$-functions lie in $\overline{\mathbb{Q}}(T)$, and $\varphi$ is good for $\rho$ iff every zero $\alpha \in \overline{\mathbb{Q}}$ of $L_{\mathcal{C}}(T, \rho \otimes \varphi)$ satisfies $|\iota(\alpha)|^{2}=1 / q^{1+w}$ for every field embedding $\iota: \overline{\mathbb{Q}} \rightarrow \mathbb{C}$. Equivalently, $\varphi$ is good for $\rho$ iff the 'unitarized' $L$-function

$$
L_{\mathcal{C}}^{*}(T, \rho \otimes \varphi):=L_{\mathcal{C}}\left(T /(\sqrt{q})^{1+w}, \rho \otimes \varphi\right)
$$

is the characteristic polynomial of a unitary conjugacy class $\theta_{\rho, q, \varphi} \subset U_{R}(\mathbb{C})$ for $R=\operatorname{deg}\left(L_{\mathcal{C}}(T, \rho)\right)$.

We further distinguish bad characters by saying that $\rho \in \Phi_{q}(c)$ is mixed for $\rho$ iff it lies in the set

$$
\Phi_{q}(c)_{\rho \text { mixed }}:=\left\{\varphi \in \Phi_{q}(c) \backslash \Phi_{q}(c)_{\rho \text { good }}: L_{\mathcal{C}}(T, \rho \otimes \varphi) \in \overline{\mathbb{Q}}_{\ell}[T]\right\},
$$

and otherwise we say that the elements of

$$
\Phi_{q}(c)_{\rho \text { heavy }}:=\Phi_{q}(c) \backslash\left(\Phi_{q}(c)_{\rho \text { good }} \cup \Phi_{q}(c)_{\rho \text { mixed }}\right)
$$

are heavy for $\rho$. The mixed characters are those for which $L_{\mathcal{C}}^{*}(T, \rho \otimes \varphi)$ is not the characteristic polynomial of a unitary matrix, and the heavy characters are those for which $L_{\mathcal{C}}^{*}(T, \rho \otimes \varphi)$ is not even a polynomial.

We are now in a position to state our main theorem: 
Theorem 2.0.8. Suppose that $\rho$ is punctually pure of weight $w$, that its Mellin transform has big monodromy (see section 3), and that $\Phi_{q}(c)_{\rho \text { heavy }} \subseteq\{\mathbf{1}\}$ for all $q$. Then

$$
\lim _{q \rightarrow \infty} \frac{\left|\Phi_{q}(c)\right|}{q^{n(1+w)}} \cdot \operatorname{Var}_{A}\left[S_{k, n, c}(A)\right]=I_{k}(n ; R)
$$

for each $n \geq 1$.

Remark 2.0.9. If $\rho$ is the representation associated to the $\ell$-adic Tate module of the Legendre curve, then the hypotheses on $c$ in Theorem 1.1.2 imply that the Mellin transform of $\rho$ has big monodromy (see [HKRG17, §8]). One can also show that $R=2 \operatorname{deg}(c)-1$ (cf. loc. cit.). Thus Theorem 1.1.2 follows from Theorem 2.0.8.

Corollary 2.0.10. In the case of $k=2$ we have for $n \leq R$

and for $R<n<2 R$

$$
\lim _{q \rightarrow \infty} \frac{\left|\Gamma_{q}(c)\right|}{q^{2 n}} \operatorname{Var}_{A}\left[S_{2, n, c}(A)\right]=\left(\begin{array}{c}
n+3 \\
3
\end{array}\right)
$$

$$
\lim _{q \rightarrow \infty} \frac{\left|\Gamma_{q}(c)\right|}{q^{2 n}} \operatorname{Var}_{A}\left[S_{2, n, c}(A)\right]=\left(\begin{array}{c}
2 R-n+3 \\
3
\end{array}\right)
$$

\section{Big Monodromy and Equidistribution}

In [HKRG17, §10], we defined a subgroup $\Phi_{q}(u)^{\nu} \subseteq \Phi_{q}(c)$, and for each coset $\varphi \Phi_{q}(u)^{\nu}$, we defined a monodromy group

$$
\mathcal{G}_{\text {geom }}\left(\varphi \Phi_{q}(u)^{\nu}, \rho \Phi_{q}(u)^{\nu}\right) \subseteq \operatorname{GL}_{R}\left(\overline{\mathbb{Q}}_{\ell}\right)
$$

generated by Frobenii corresponding to the good characters in $\varphi \Phi_{q}(u)^{\nu}$, where $R:=\operatorname{deg}\left(L_{\mathcal{C}}(T, \rho)\right)=$ $\operatorname{deg}\left(L_{\mathcal{C}}(T, \rho \otimes \varphi)\right)$ (see HKRG17, Prop. 4.3.1]). More precisely, the Frobenius attached to a character $\varphi \alpha^{\nu} \in \varphi \Phi_{q}(u)^{\nu}$ is an element of $\mathrm{GL}_{R}\left(\overline{\mathbb{Q}}_{\ell}\right)$ with characteristic polynomial $L_{\mathcal{C}}\left(T, \rho \otimes \chi \alpha^{\nu}\right)$, and $\mathcal{G}_{\text {geom }}\left(\varphi \Phi_{q}(u)^{\nu}, \rho \Phi_{q}(u)^{\nu}\right)$ is the Zariski closure of all such elements when one takes $q \rightarrow \infty$.

We say that $\varphi \in \Phi_{q}(c)$ is big for $\rho$ iff it lies in the set

$$
\Phi_{q}(c)_{\rho \text { big }}:=\left\{\varphi \in \Phi_{q}(c): \mathcal{G}_{\text {geom }}\left(\varphi \Phi_{q}(u)^{\nu}, \rho \Phi_{q}(u)^{\nu}\right)=\operatorname{GL}_{R}\left(\overline{\mathbb{Q}}_{\ell}\right)\right\},
$$

and we say that the Mellin transform of $\rho$ has big monodromy iff

$$
\left|\Phi_{q}(c)\right| \sim\left|\Phi_{q}(c)_{\rho \text { big }}\right| \text { as } q \rightarrow \infty .
$$

Lemma 3.0.1. If the Mellin transform of $\rho$ has big monodromy, then

$$
\left|\Phi_{q}(c)\right| \sim\left|\Phi_{q}(c)_{\rho \text { good }}\right| \sim\left|\Phi_{q}(c)_{\rho \text { big }}\right| \text { as } q \rightarrow \infty
$$

Proof. See [HKRG17, Corollary . 10.4.3].

If the Mellin transform of $\rho$ has big monodromy it implies that $\theta_{\rho, q, \varphi}$ become equidistributed in $U_{R}(\mathbb{C})$ (for reference see Theorem 10.0.4 combined with remark 8.2.4 in [HKRG17]):

Theorem 3.0.2. Suppose that $\rho$ is punctually pure of weight $w$, that its Mellin transform has big monodromy, and that $\Phi_{q}(c)_{\rho \text { heavy }} \subseteq\{\mathbf{1}\}$ for all $q$. Then for any continuous function $F: U_{R}(\mathbb{C}) \rightarrow \mathbb{C}$

$$
\lim _{q \rightarrow \infty} \frac{1}{\left|\Phi_{q}(c)_{\rho \text { good }}\right|} \sum_{\varphi \in \Phi_{q}(c)_{\rho \text { good }}} F\left(\theta_{\rho, q, \varphi}\right)=\int_{U_{R}(\mathbb{C})} F(\theta) d \theta
$$

with respect to Haar measure $d \theta$ on $U_{R}(\mathbb{C})$.

In the following Theorem we present a sufficient criteria for the Mellin transform of $\rho$ to have big monodromy (explicit example for representations meeting this criteria can be found in [HKRG17, $\S 12])$. 
Theorem 3.0.4. Let $s$ be the conductor of $\rho$ and suppose that $\operatorname{gcd}(s, c)=t$ and that $\operatorname{deg} c \geq 3$. Suppose moreover that $V(0)$ has a unique unipotent block of exact multiplicity one and that $\rho$ is geometrically simple and pointwise pure. If $r:=\operatorname{dim}(V)$ and $\operatorname{deg} c$ satisfy

$$
\operatorname{deg} c>\frac{1}{r}\left(72\left(r^{2}+1\right)^{2}-r-\operatorname{deg}(L(T, \rho))+\sum_{\nu \in C} \operatorname{deg} \nu\left(r-\operatorname{deg} L\left(T, \rho_{\nu}\right)\right)\right.
$$

then the Mellin transform of $\rho$ has big monodromy.

\section{Proof of Theorem 2.0.8}

We express the variance of the arithmetic progressions sums $S_{k, n, c}(A)$ in terms of sums of divisor functions twisted by Dirichlet characters. Let $k$ be a positive integer and let

$$
c_{k, \rho \otimes \varphi, n}=\sum_{\substack{f \in \mathcal{M}_{n} \\ \operatorname{gcd}(f, c)=1}} d_{k, \rho \otimes \varphi}(f)
$$

be the coefficients in the expansion of the $\mathrm{k}$-th power of the partial L-function (2.0.4)

$$
L_{\mathcal{C}}(T, \rho \otimes \varphi)^{k}=\sum_{n=0}^{\infty} c_{k, \rho \otimes \varphi, n} T^{n}
$$

For each $\varphi \in \Phi_{q}(c)$, we extend $\varphi$ to a multiplicative map $\varphi_{!}: \mathcal{M} \rightarrow \overline{\mathbb{Q}}_{\ell}$ by defining:

$$
\varphi_{!}(f)= \begin{cases}\varphi\left(f+c \mathbb{F}_{q}[t]\right) & \text { if } \operatorname{gcd}(f, c)=1 \\ 0 & \text { otherwise }\end{cases}
$$

It is multiplicative and satisfies

$$
\varphi_{!}(\pi)=\left\{\begin{array}{ll}
\varphi\left(\operatorname{Frob}_{v(\pi)}\right) & \text { if } \pi \nmid c \\
0 & \text { otherwise }
\end{array} \text { for } \pi \in \mathcal{I} .\right.
$$

The Orthogonality relations for Dirichlet characters are:

(i) for each $A_{1}, A_{2} \in \Gamma_{q}(c)$

$$
\frac{1}{\left|\Phi_{q}(c)\right|} \sum_{\varphi \in \Phi_{q}(c)} \varphi_{!}\left(A_{1}\right) \bar{\varphi}_{!}\left(A_{2}\right)= \begin{cases}1 & \text { if } A_{1}=A_{2} \bmod c \\ 0 & \text { if otherwise }\end{cases}
$$

(ii) for every $\varphi_{1}, \varphi_{2} \in \Phi_{q}(c)$

$$
\frac{1}{\left|\Phi_{q}(c)\right|} \sum_{A \in \Gamma_{q}(c)} \varphi_{1 !}(A) \bar{\varphi}_{2 !}(A)= \begin{cases}1 & \text { if } \varphi_{1}=\varphi_{2} \\ 0 & \text { if } \varphi_{1} \neq \varphi_{2}\end{cases}
$$

We use (4.0.2) and (4.0.1) to express the sum of $d_{k, \rho}(f)$ over arithmetic progressions in terms of $c_{k, \rho \otimes \varphi, n}$ :

$$
S_{n, c, q}(A)=\frac{1}{\left|\Phi_{q}(c)\right|} \sum_{f \in \mathcal{M}_{n}} d_{k, \rho}(f) \sum_{\varphi \in \Phi_{q}(c)} \varphi_{!}(A) \bar{\varphi} !(f)=\frac{1}{\left|\Phi_{q}(c)\right|} \sum_{\varphi \in \Phi_{q}(c)} c_{k, \rho \otimes \varphi, n} \cdot \bar{\varphi}(A)
$$


Therefore, if we write $\mathbf{1} \in \Phi_{q}(c)$ for the trivial character and by using the second orthogonality relation (4.0.3) then expected value of $S_{n, c, q}(A)$ equals

$$
\begin{aligned}
\mathbb{E}_{A}\left[S_{n, c, q}(A)\right] & :=\frac{1}{\left|\Phi_{q}(c)\right|} \sum_{A \in \Gamma_{q}(c)} S_{n, c, q}(A) \\
& =\frac{1}{\left|\Phi_{q}(c)\right|^{2}} \sum_{\varphi \in \Phi_{q}(c)} c_{k, \rho \otimes \varphi, n} \sum_{A \in \Gamma_{q}(c)} \bar{\varphi}_{!}(A) \\
& =\frac{1}{\left|\Phi_{q}(c)\right|} c_{k, \rho \otimes \mathbf{1}, n}
\end{aligned}
$$

In particular, we have the identity

$$
S_{n, c, q}(A)-\mathbb{E}_{A}\left[S_{n, c, q}(A)\right]=\frac{1}{\left|\Phi_{q}(c)\right|} \sum_{\substack{\varphi \in \Phi_{q}(c) \\ \varphi \neq 1}} c_{k, \rho \otimes \varphi, n} \cdot \bar{\varphi}(A) .
$$

Now consider the variance

$$
\operatorname{Var}_{A}\left[S_{n, c, q}(A)\right]=\frac{1}{\left|\Phi_{q}(c)\right|} \sum_{A \in \Gamma_{q}(c)}\left|S_{n, c, q}(A)-\mathbb{E}_{A}\left[S_{n, c, q}(A)\right]\right|^{2} .
$$

If we apply identities (4.0.3) and (4.0.5), then the right side equals

$$
\frac{1}{\left|\Phi_{q}(c)\right|^{3}} \sum_{A \in \Gamma_{q}(c)} \sum_{\substack{\varphi_{1}, \varphi_{2} \in \Phi_{q}(c) \\ \varphi_{1 !}, \varphi_{2} ! \neq 1}} c_{k, \rho \otimes \varphi_{1}, n} \overline{c_{k, \rho \otimes \varphi_{2}, n}} \cdot \bar{\varphi}_{1 !}(A) \varphi_{2 !}(A)=\frac{1}{\left|\Phi_{q}(c)\right|^{2}} \sum_{\substack{\varphi \in \Phi_{q}(c) \\ \varphi \neq \mathbf{1}}}\left|c_{k, \rho \otimes \varphi, n}\right|^{2} .
$$

In summary, the function $S_{n, c, q}(A)$ of the random variable $A$ satisfies

$$
\mathbb{E}_{A}\left[S_{n, c, q}(A)\right]=\frac{1}{\left|\Phi_{q}(c)\right|} c_{k, \rho \otimes \mathbf{1}, n}, \quad \operatorname{Var}_{A}\left[S_{n, c, q}(A)\right]=\frac{1}{\left|\Phi_{q}(c)\right|^{2}} \sum_{\substack{\varphi \in \Phi_{q}(c) \\ \varphi \neq \mathbf{1}}}\left|c_{k, \rho \otimes \varphi, n}\right|^{2}
$$

Next, we break $c_{k, \rho \otimes \varphi, n}$ into smaller pieces which we will express in terms of the associated Frobenius matrices:

\section{Lemma 4.0.7.}

$$
c_{k, \rho \otimes \varphi, n}=\sum_{n_{1}+\cdots+n_{k}=n} \prod_{i=1}^{k} c_{1, \rho \otimes \varphi, n_{i}}
$$

Proof. This follows immediately from the definition of $c_{k, \rho \otimes \varphi, n}$ as the coefficients in the expansion of $L_{\mathcal{C}}(T, \rho \otimes \varphi)^{k}$ :

$$
L_{\mathcal{C}}(T, \rho \otimes \varphi)^{k}=\left(\sum_{n=0}^{\infty} c_{1, \rho \otimes \varphi, n} T^{n}\right)^{k}=\sum_{n=0}^{\infty} \sum_{n_{1}+\ldots+n_{k}=n} \prod_{i=1}^{k} c_{1, \rho \otimes \varphi, n_{i}} T^{n}
$$

Recall that std: $U_{R}(\mathbb{C}) \rightarrow \mathrm{GL}_{R}(\mathbb{C})$ is the representation given by the inclusion $U_{R}(\mathbb{C}) \subset \mathrm{GL}_{R}(\mathbb{C})$ and

$$
\wedge^{j} \operatorname{std}: U_{R}(\mathbb{C}) \rightarrow \mathrm{GL}_{R_{j}}\left(\wedge^{j} \mathbb{C}\right)
$$

is its $j$ th exterior power where $R_{j}=\left(\begin{array}{c}R \\ j\end{array}\right)$; and we defined $\left(\wedge^{j} \operatorname{std}\right)(g)=0$ unless $0 \leq j \leq R$.

Lemma 4.0.9. Let $\varphi \in \Phi_{q}(c)_{\rho \text { good }}$, then

$$
c_{1, \rho \otimes \varphi, n}=(-1)^{n} \cdot(\sqrt{q})^{n(1+w)} \cdot \operatorname{Tr}\left(\left(\wedge^{n} \operatorname{std}\right)\left(\theta_{\rho, q, \varphi}\right)\right)
$$


Proof. A Dirichlet character $\varphi$ is good for $\rho$ iff the 'unitarized' $L$-function

$$
L_{\mathcal{C}}^{*}(T, \rho \otimes \varphi):=L_{\mathcal{C}}\left(T /(\sqrt{q})^{1+w}, \rho \otimes \varphi\right)
$$

is the characteristic polynomial of a unitary conjugacy class $\theta_{\rho, q, \varphi} \subset U_{R}(\mathbb{C})$ for $R=\operatorname{deg}\left(L_{\mathcal{C}}(T, \rho)\right)$.

The coefficients of the characteristic polynomial of an $N \times N$ matrix with eigenvalues $\lambda_{1}, \ldots, \lambda_{n}$ are the elementary symmetric functions $\sum_{1 \leq i_{1}<\ldots<i_{r}} \lambda_{i_{1}} \cdots \lambda_{i_{r}}$ which give the character of the exterior power representation. Thus we may write

$$
L_{\mathcal{C}}^{*}(T, \rho \otimes \varphi)=\sum_{i=0}^{R}(-1)^{i} \cdot(\sqrt{q})^{i(1+w)} \cdot \operatorname{Tr}\left(\left(\wedge^{i} \operatorname{std}\right)\left(\theta_{\rho, q, \varphi}\right)\right) T^{i}
$$

Lemma 4.0.10. If $\varphi \in \Phi_{q}(c)$, then

$$
\left|c_{1, \rho \otimes \varphi, n}\right|^{2}= \begin{cases}O\left(q^{n(2+w)}\right) & \text { if } \varphi \in \Phi_{q}(c)_{\rho \text { heavy }} \\ O\left(q^{n(1+w)}\right) & \text { otherwise }\end{cases}
$$

The implied constants depend only on $\rho$ and $n$.

Proof. Fix a field embedding $\iota: \overline{\mathbb{Q}} \rightarrow \mathbb{C}$ and identify $\overline{\mathbb{Q}}$ with its image. Recall that, for some integer $s$ depending on $\varphi$ and satisfying $s \leq \operatorname{dim}(V)$, we can express $L_{\mathcal{C}}(T, \rho \otimes \varphi)$ as a ratio

$$
\prod_{i=1}^{s+R}\left(1-\alpha_{i} T\right) / \prod_{j=1}^{s}\left(1-\beta_{j} T\right)
$$

where the $\alpha_{i}$ and $\beta_{j}$ lie in $\overline{\mathbb{Q}}$ and satisfy

$$
\left|\alpha_{i}\right|^{2} \leq q^{1+w}, \quad\left|\beta_{j}\right|^{2} \leq q^{2+w} .
$$

For reference see equation (3.4.2) and Theorem 6.2.1 in [HKRG17]. If $\varphi \notin \Phi_{q}(c)_{\rho \text { heavy }}$, then $s=0$ and $\left|\alpha_{i}\right|^{2} \leq q^{1+w}$, and thus

$$
\left|c_{1, \rho \otimes \varphi, n}\right|^{2}=\left|\sum_{i_{1}<\cdots<i_{n}} \alpha_{i_{1}} \cdots \alpha_{i_{n}}\right|^{2} \leq \sum_{i_{1}<\cdots<i_{n}}\left|\alpha_{i_{1}} \cdots \alpha_{i_{n}}\right|^{2} \leq\left(\begin{array}{c}
R \\
k
\end{array}\right) q^{1+w} .
$$

When $\rho$ is heavy we get

$$
\begin{aligned}
\left|c_{1, \rho \otimes \varphi, n}\right|^{2} & =\left|\sum_{n_{1}+n_{2}=n}(-1)^{n_{1}} \sum_{i_{1}<\cdots<i_{n_{1}}} \alpha_{i_{1}} \cdots \alpha_{i_{n_{1}}} \sum_{j_{1} \leq \cdots \leq j_{n_{2}}} \beta_{j_{1}} \cdots \beta_{j_{n_{2}}}\right|^{2} \\
& \leq \sum_{n_{1}+n_{2}=n} \sum_{i_{1}<\cdots<i_{n_{1}}} \sum_{j_{1} \leq \cdots \leq j_{n_{2}}}\left|\alpha_{i_{1}} \cdots \alpha_{i_{n}} \beta_{j_{1}} \cdots \beta_{j_{n_{2}}}\right|^{2} \\
& =O\left(q^{n(2+w)}\right) .
\end{aligned}
$$

Corollary 4.0.11. If $\varphi \in \Phi_{q}(c)$, then

$$
\left|c_{k, \rho \otimes \varphi, n}\right|^{2}= \begin{cases}O\left(q^{n(2+w)}\right) & \text { if } \varphi \in \Phi_{q}(c)_{\rho \text { heavy }} \\ O\left(q^{n(1+w)}\right) & \text { otherwise }\end{cases}
$$

The implied constants depend only on $\rho$ and $n$.

Proof. Combine Lemma 4.0.7 and Lemma 4.0.10. 
Lemma 4.0.12. If the Mellin transform of $\rho$ has big monodromy and if $\Phi_{q}(c)_{\rho \text { heavy }} \subseteq\{\mathbf{1}\}$, then

$$
\sum_{\substack{\varphi \in \Phi_{q}(c) \\ \varphi \neq \mathbf{1}}}\left|c_{k, \rho \otimes \varphi, n}\right|^{2} \sim \sum_{\substack{\varphi \in \Phi_{q}(c)_{\rho \text { good }} \\ \varphi \neq \mathbf{1}}}\left|c_{k, \rho \otimes \varphi, n}\right|^{2} \quad \text { as } q \rightarrow \infty
$$

Proof. We break the sum over all Dirichlet characters mod $c$ to sums over "good", "heavy" and "mixed" characters (see definitions (2.0.5), (2.0.7), (2.0.6) )

$$
\sum_{\substack{\varphi \in \Phi_{q}(c) \\ \varphi \neq \mathbf{1}}}\left|c_{k, \rho \otimes \varphi, n}\right|^{2}=\sum_{\substack{\varphi \in \Phi_{q}(c)_{\rho} \text { good } \\ \varphi \neq \mathbf{1}}}\left|c_{k, \rho \otimes \varphi, n}\right|^{2}+\sum_{\substack{\varphi \in \Phi_{q}(c)_{\rho} \text { heavy } \\ \varphi \neq \mathbf{1}}}\left|c_{k, \rho \otimes \varphi, n}\right|^{2}+\sum_{\substack{\varphi \in \Phi_{q}(c)_{\rho \text { mixed }} \\ \varphi \neq \mathbf{1}}}\left|c_{k, \rho \otimes \varphi, n}\right|^{2}
$$

which give by Corollary 4.0.11

$$
\sum_{\substack{\varphi \in \Phi_{q}(c)_{\rho \text { good }} \\ \varphi \neq \mathbf{1}}}\left|c_{k, \rho \otimes \varphi, n}\right|^{2}+\left|\Phi_{q}(c)_{\rho \text { heavy }} \backslash\{1\}\right| \cdot O\left(q^{n(2+w)}\right)+\left|\Phi_{q}(c)_{\rho \text { mixed }} \backslash\{1\}\right| \cdot O\left(q^{n(1+w)}\right)
$$

Lemma 3.0.1 and having $\Phi_{q}(c)_{\rho \text { heavy }} \subseteq\{\mathbf{1}\}$ conclude the proof.

Proposition 4.0.13. If the Mellin transform of $\rho$ has big monodromy and if $\Phi_{q}(c)_{\rho \text { heavy }} \subseteq\{\mathbf{1}\}$, then

$$
\lim _{q \rightarrow \infty} \frac{\left|\Phi_{q}(c)\right|}{q^{n(1+w)}} \cdot \operatorname{Var}_{A}\left[S_{n, c, q}(A)\right]=\int_{U_{R}(\mathbb{C})}\left|\sum_{\substack{n_{1}+\cdots+n_{k}=n \\ 0 \leq n_{1}, \ldots, n_{k} \leq R}} \operatorname{Tr}\left(\left(\otimes_{i=1}^{k}\left(\wedge^{n_{i}} \operatorname{std}\right)\right)(\theta)\right)\right|^{2} d \theta .
$$

Proof. In (4.0.6) we found the following expression for the variance

$$
\operatorname{Var}_{A}\left[S_{n, c, q}(A)\right]=\frac{1}{\left|\Phi_{q}(c)\right|^{2}} \sum_{\substack{\varphi \in \Phi_{q}(c) \\ \varphi \neq 1}}\left|c_{k, \rho \otimes \varphi, n}\right|^{2} .
$$

In the limit of $q \rightarrow \infty$ we have from Lemma 4.0 .12

$$
\operatorname{Var}_{A}\left[S_{n, c, q}(A)\right] \sim \frac{1}{\left|\Phi_{q}(c)\right|^{2}} \sum_{\substack{\varphi \in \Phi_{q}(c)_{\rho \text { good }} \\ \varphi \neq \mathbf{1}}}\left|c_{k, \rho \otimes \varphi, n}\right|^{2}
$$

which Lemma 4.0.7 and Lemma 4.0.9 equals to

$$
\frac{1}{\left|\Phi_{q}(c)\right|^{2}} \sum_{\substack{\varphi \in \Phi_{q}(c) \rho \text { good } \\ \varphi \neq \mathbf{1}}}\left|\sum_{\substack{n_{1}+\cdots+n_{k}=n \\ 0 \leq n_{1}, \ldots, n_{k} \leq R}} \operatorname{Tr}\left(\left(\otimes_{i=1}^{k}\left(\wedge^{n_{i}} \mathrm{std}\right)\right)\left(\theta_{\rho, q, \varphi}\right)\right)\right|^{2}
$$

Now note that $\left|\Phi_{q}(c)\right| \sim\left|\Phi_{q}(c)_{\rho \text { good }}\right|$ (Lemma 3.0.1) and apply the equidistribution result Theorem 3.0 .2 to conclude the proof.

\section{Matrix Integral}

In this section we state a few results evaluating the matrix integral in certain ranges and its asymptotic behaviour. Proofs can be found in [KRRGR18].

In certain ranges the matrix integral evaluates to a very simple expression. For $n<R$ or $(k-1) R<n<k R$, we obtain the following formulae:

Theorem 5.0.1. Let $I_{k}(n ; R)$ be the matrix integral defined in (1.1.1). Then 
(i) for $(k-1) R<n<k R$,

$$
I_{k}(n ; R)=\left(\begin{array}{c}
k R-n+k^{2}-1 \\
k^{2}-1
\end{array}\right)
$$

(ii) for $n<R$

$$
I_{k}(n ; R)=\left(\begin{array}{c}
n+k^{2}-1 \\
k^{2}-1
\end{array}\right)
$$

There is also a closed-form formula for the matrix integral for any range of the parameters, in terms of a lattice point count:

Theorem 5.0.2. $I_{k}(m ; N)$ is equal to the count of lattice points $x=\left(x_{i}^{(j)}\right) \in \mathbb{Z}^{k^{2}}$ satisfying each of the following relations:

(i) $0 \leq x_{i}^{(j)} \leq N$ for all $1 \leq i, j \leq k$;

(ii) $x_{1}^{(k)}+x_{2}^{(k-1)}+\cdots+x_{k}^{(1)}=k N-m$, and

(iii) $x$ is a $k \times k$ matrix whose entries satisfy the following system of inequalities,

$$
\begin{aligned}
& x_{1}^{(1)} \leq x_{1}^{(2)} \leq \cdots \leq x_{\vee}^{(k)} \\
& x_{2}^{(1)} \leq x_{2}^{(2)} \leq \cdots \leq x_{2}^{(k)} \\
& \begin{array}{cccc}
\vdots & \vdots & \ddots & \vdots \\
\vee \mathrm{VI} & \mathrm{VI} & & \mathrm{VI}
\end{array} \\
& x_{k}^{(1)} \leq x_{k}^{(2)} \leq \cdots \leq x_{k}^{(k)}
\end{aligned}
$$

The asymptotic behavior of $I_{k}(n ; R)$ for $n \approx R$ is given in the following theorem:

Theorem 5.0.3. Let $c:=n / R$. Then for $c \in[0, k]$,

$$
I_{k}(n ; R)=\gamma_{k}(c) R^{k^{2}-1}+O_{k}\left(R^{k^{2}-2}\right),
$$

with

$$
\gamma_{k}(c)=\frac{1}{k ! G(1+k)^{2}} \int_{[0,1]^{k}} \delta_{c}\left(w_{1}+\ldots+w_{k}\right) \prod_{i<j}\left(w_{i}-w_{j}\right)^{2} d^{k} w,
$$

with $\delta_{c}(x)=\delta(x-c)$ being the delta distribution translated by $c$, and $G$ is the Barnes $G$-function, so that for positive integers $k, G(1+k)=1 ! \cdot 2 ! \cdot 3 ! \cdots(k-1) !$.

\section{REFERENCES}

[Blo08] V. Blomer, The average value of divisor sums in arithmetic progressions, Q. J. Math. 59 (2008), no. 3, 275-286. MR2444061 愢

$\left[\mathrm{CFK}^{+} 05\right]$ J. B. Conrey, D. W. Farmer, J. P. Keating, M. O. Rubinstein, and N. C. Snaith, Integral moments of L-functions, Proc. London Math. Soc. (3) 91 (2005), no. 1, 33-104. MR2149530 11

[Cra22] Harald Cramér, über zwei Sätze des Herrn G. H. Hardy, Math. Z. 15 (1922), no. 1, 201-210. MR1544568 11

[Del80] Pierre Deligne, La conjecture de Weil. II, Inst. Hautes Études Sci. Publ. Math. 52 (1980), $137-252$. MR601520 12

[FKM15] Étienne Fouvry, Emmanuel Kowalski, and Philippe Michel, On the exponent of distribution of the ternary divisor function, Mathematika 61 (2015), no. 1, 121-144. MR3333965 11

[HB92] D. R. Heath-Brown, The distribution and moments of the error term in the Dirichlet divisor problem, Acta Arith. 60 (1992), no. 4, 389-415. MR1159354 任 
[HKRG17] Chris Hall, Jonathan P. Keating, and Edva Roditty-Gershon, Variance of sums in arithmetic progressions of arithmetic functions associated with higher degree l-functions in $\mathbb{F}_{q}[t]$, arXiv:1703.09190v1 (2017). 12 . 2.0.9, 3, 3, 目

[KR14] Emmanuel Kowalski and Guillaume Ricotta, Fourier coefficients of $G L(N)$ automorphic forms in arithmetic progressions, Geom. Funct. Anal. 24 (2014), no. 4, 1229-1297. MR3248485 11

[KRRGR18] J. P. Keating, B. Rodgers, E. Roditty-Gershon, and Z. Rudnick, Sums of divisor functions in $\mathbb{F}_{q}[t]$ and matrix integrals, Math. Z. 288 (2018), no. 1-2, 167-198. MR3774409 11 1 1 5

[LZ12] Yuk-Kam Lau and Lilu Zhao, On a variance of Hecke eigenvalues in arithmetic progressions, J. Number Theory 132 (2012), no. 5, 869-887. MR2890517 11

[Mot73] Yoichi Motohashi, On the distribution of the divisor function in arithmetic progressions, Acta Arith. 22 (1973), 175-199. MR0340196 11

[RS17] Brad Rodgers and Kannan Soundararajan, The variance of divisor sums in arithmetic progressions, arXiv:1610.06900v2 (2017). 11

[Tit86] E. C. Titchmarsh, The theory of the Riemann zeta-function, Second, The Clarendon Press, Oxford University Press, New York, 1986. Edited and with a preface by D. R. Heath-Brown. MR882550 11

[Ton56] Kwang-Chang Tong, On divisor problems. II, III, Acta Math. Sinica 6 (1956), 139-152, 515-541. MR0098718 愢

DEPARTMENT OF MATHEMATICS, WESTERN UNIVERSITY, LONDON, ON, CANADA, N6A 5B7

SCHOOL OF MATHEMATICS, UNIVERSITY OF BRISTOL, BRISTOL BS8 1TW, UK

SCHOOL OF MATHEMATICS, UNIVERSITY OF BRISTOL, BRISTOL BS8 1TW, UK 